\title{
Topological susceptibility, divergent chiral density, and phase diagram of chirally imbalanced QCD medium at finite temperature
}

\author{
Marco Ruggieri, ${ }^{1, *}$ Maxim N. Chernodub $\odot{ }^{2,3, \uparrow}$ and Zhen-Yan Lu $\oplus^{4, \$}$ \\ ${ }^{1}$ School of Nuclear Science and Technology, Lanzhou University, \\ 222 South Tianshui Road, Lanzhou 730000, China \\ ${ }^{2}$ Institut Denis Poisson UMR 7013, Université de Tours, 37200 France \\ ${ }^{3}$ Pacific Quantum Center, Far Eastern Federal University, Sukhanova 8, Vladivostok, 690950, Russia \\ ${ }^{4}$ School of Physics and Electronic Science, Hunan University of Science and Technology, \\ Xiangtan 411201, China
}

(Received 25 April 2020; accepted 7 July 2020; published 17 July 2020)

\begin{abstract}
We show that the nonlocal two-flavor Nambu-Jona-Lasinio model predicts the enhancement of both chiral and axial symmetry breaking as the chiral imbalance of hot QCD matter, regulated by a chiral chemical potential $\mu_{5}$, increases. The two crossovers are reasonably close to each other in the range of $\mu_{5}$ examined here, and the pseudocritical temperatures rise with $\mu_{5}$. The curvatures of the chiral and axial crossovers for the chiral quark chemical potential approximately coincide and give $\kappa_{5} \simeq-0.011$. We point out that the presence of $\mu_{5}$ in thermodynamic equilibrium is inconsistent with the fact that the chiral charge is not a Noetherconserved quantity for massive fermions. The chiral chemical potential should not, therefore, be considered as a true chemical potential that sets a thermodynamically stable environment in the massive theory, but rather as a new coupling that may require a renormalization in the ultraviolet domain. The divergence of an unrenormalized chiral density, coming from zero-point fermionic fluctuations, is a consequence of this property. We propose a solution to this problem via a renormalization procedure.
\end{abstract}

DOI: 10.1103/PhysRevD.102.014031

\section{INTRODUCTION}

The chirality plays an important role in the fundamental theory of strong interactions of quarks and gluons described by QCD. In QCD with $N_{f}$ massless quarks, the classical formulation of QCD enjoys the invariance under $S U\left(N_{f}\right)_{L / R}$ chiral transformations $\psi_{L / R} \rightarrow U_{L / R} \psi_{L / R}$, which transforms, separately, the $N_{f}$-plets of quarks with left-handed, $\psi_{L}$, and right-handed, $\psi_{R}$, chiralities. As a consequence of the Noether theorem, the chiral charge is a conserved quantity at the level of classical equations of motion. In the quantum version of $\mathrm{QCD}$, the chirality is no longer a conserved number because quantum fluctuations break the chiral symmetry spontaneously. The chiral condensate, $\langle\bar{\psi} \psi\rangle \equiv\left\langle\bar{\psi}_{L} \psi_{R}\right\rangle+\left\langle\bar{\psi}_{R} \psi_{L}\right\rangle$, dynamically breaks the full chiral group down to its diagonal (vector) subgroup,

\footnotetext{
*uggieri@lzu.edu.cn

†maxim.chernodub@idpoisson.fr

*luzhenyan@hnust.edu.cn
}

Published by the American Physical Society under the terms of the Creative Commons Attribution 4.0 International license. Further distribution of this work must maintain attribution to the author(s) and the published article's title, journal citation, and DOI. Funded by SCOAP.
$S U\left(N_{f}\right)_{L} \times S U\left(N_{f}\right)_{R} \equiv S U\left(N_{f}\right)_{V} \times S U\left(N_{f}\right)_{A} \rightarrow S U\left(N_{f}\right)_{V}$. Given the number of the light quarks, $N_{f}=2$, the chiral symmetry breaking manifests itself in the appearance of three Goldstone bosons, the light pseudoscalar mesons, that correspond to the number of spontaneously broken generators, $2 N_{f}-1$, of the chiral $S U\left(N_{f}\right)_{A}$ subgroup [1]. At a classical level, QCD also possesses the axial symmetry which reflects the invariance of the QCD Lagrangian under the axial $U(1)_{A}$ transformations that rotate all quark flavors by the same, chirality-sensitive phase: $\psi_{L / R} \rightarrow e^{ \pm i \omega} \psi_{L / R}$. This symmetry is broken at the quantum level by the axial anomaly, which leads to nonconservation of the (otherwise, classically conserved) axial current. The chiral and axial symmetries of quarks are intrinsically related to the topological properties of the vacuum which are determined by the gluonic sector of the theory [1].

It is established that at zero baryon chemical potential the QCD medium experiences a smooth crossover from the hadronic, low-temperature phase characterized by a nonzero chiral condensate to the high-temperature phase of quark-gluon plasma where the chiral condensate is almost vanishing. As a result, the chiral symmetry gets approximately restored with the rise of the temperature. The fact that this is a crossover rather than a transition is related to the nonzero quark masses that break chiral symmetry 
explicitly, so the chiral condensate serves as an approximate order parameter of the chiral symmetry breaking. There are also arguments as to why the axial symmetry should also be restored at high temperature [2]. The nonconservation of the axial current is related to the presence of topologically nontrivial configurations, usually associated with the instantons. The strength of the instanton fluctuations, quantitatively determined by the topological susceptibility, depends on the environment: at high temperatures, instantons-based interactions among quarks are suppressed. Therefore, one expects that the topological susceptibility is small at high temperatures and the axial symmetry, similarly to the chiral symmetry, gets restored at the high-temperature phase. So far, a clear relation between chiral and axial symmetry restoration is not very clear. Although the topological susceptibility is not an order parameter, it seems natural to consider this to be a relevant quantity for quantifying the axial symmetry breaking and to define the crossover for axial symmetry restoration as the temperature range in which the topological susceptibility has the largest change with temperature. This is the strategy that we adopt in the present article, in which we report on the study of the restoration of chiral and axial symmetry, studying the latter by means of the topological susceptibility.

In this article, we model a thermal QCD medium at finite chiral chemical potential, $\mu_{5}$, the latter being conjugated to the asymmetry between left- and right-handed particles density, $n_{5}$. The chiral sector of QCD affects also various transport properties of the system due to the presence of the axial anomaly. The most famous example of such phenomena is the chiral magnetic effect (CME) which generates an electric current [3-8]: $\langle\boldsymbol{J}\rangle=\left(e^{2} / 2 \pi^{2}\right) \mu_{5} \boldsymbol{B}$, in the presence of an external magnetic field $\boldsymbol{B}$ in a system of massless fermions possessing a nonzero chiral density $n_{5} \neq 0$. The chiral density corresponds to the difference in densities of quarks with right- and left-handed chiralities, $n_{5}=n_{R}-n_{L}$, encoded in the difference between their chemical potentials, $\mu_{5}=\mu_{L}-\mu_{R}$. The chiral chemical potential $\mu_{5}$ is thermodynamically conjugated to the chiral density $n_{5}$, so that in the thermal equilibrium, the finite density of massless fermions is set by the help of the matter source term $\delta L=\mu_{5} n_{5}$. The chiral density is the temporal component of the chiral (axial) 4-current:

$$
j_{5}^{\mu} \equiv\left(n_{5}, j_{5}\right)=\bar{\psi} \gamma^{\mu} \gamma^{5} \psi
$$

The CME is suggested to play an essential role in a wide number of physical systems ranging from astrophysical systems and quark-gluon plasmas to chiral materials [9]. Although the CME does not exist in a thermodynamic equilibrium [10-15], the electric CME current is a nondissipative quantity even in the presence of strong interactions thanks to the topological protection [9]. In the electromagnetic sector, the nonzero chiral density $\left(\mu_{5} \neq 0\right)$ may be induced via the axial anomaly that creates an imbalance in densities between right- and left-handed chiral quarks in the near-equilibrium background of parallel electric $\boldsymbol{E}$ and magnetic $\boldsymbol{B}$ fields. In the context of QCD, the chiral density may also appear in the gluon sector, due to topological transitions between different vacuum states mediated by the instanton or sphaleron phase transitions. The chiral density depletes due to mass effects, pion and sigma exchanges at low temperature [16], and the Compton scattering at high temperature [17]. Regardless of the microscopic processes involved, the use of $\mu_{5}$ is appropriate as long as one considers a thermodynamic system on a timescale larger than that of the equilibration time.

In this study, the interaction among quarks is mimicked by a nonlocal Nambu-Jona-Lasinio (NJL) model [18-34]. The nonlocal NJL models have been revealed to be appropriate for the study of the chiral medium at finite temperature, in particular because they predict that the critical temperature for chiral symmetry restoration, $T_{c}$, increases with $\mu_{5}[35,36]$, differently from what has been found within models with a local interaction kernel [7,37-40] (see, however, Ref. [41]) and in agreement with lattice simulations [42-44] and calculations based on Schwinger-Dyson equations [45-47], see also [48-54] for other recent literature on the subject.

We report on the calculation of $T_{c}$ versus $\mu_{5}$, confirming the previous findings that $T_{c}$ increases with the chiral chemical potential at least as long as $\mu_{5}$ is smaller than the typical ultraviolet scale of the model (for large values of $\mu_{5}$, one should include the backreaction on the interaction kernel, but the computation of this is well beyond the scope of our study). Part of the study presented here is devoted to the divergence of $n_{5}$ coming from ultraviolet fermion modes' we discuss how this divergence arises from the tail of the quark mass function and how this divergence can be cured via a renormalization procedure. Then, we compute the topological susceptibility as a function of $T$ and $\mu_{5}$ and try to relate this to a possible crossover from a low-temperature phase in which axial symmetry is broken by axions to the high-temperature phase in which axial symmetry is partly restored; we also comment on the simultaneity of this crossover and the chiral one, finding that the two have substantial overlap, suggesting that restoration of chiral symmetry is accompanied by the restoration of the axial symmetry.

The structure of this paper is as follows. In Sec. II, we present the nonlocal NJL model used in the study, discussing also a renormalization of $n_{5}$. In Sec. III, we present our results about chiral and axial symmetry at finite temperature and $\mu_{5}$. Finally, in Sec. IV, we collect our conclusions.

\section{MODEL}

\section{A. Thermodynamic potential}

In this work, we use a nonlocal version of the NJL model [20]. As one of the main goals of this work is to evaluate the 
topological susceptibility, it is necessary to introduce the $\theta$ dependence of the thermodynamic potential. In the QCD Lagrangian, the topological angle $\theta$ appears as a $C P$-odd term for the gluon fields,

$$
\delta \mathcal{L}_{\theta}=\theta Q,
$$

where the topological number of the gluonic field configuration is computed via the field-strength tensor $F_{\mu \nu}^{a}$ :

$$
\begin{aligned}
Q \equiv \Delta N_{\mathrm{CS}} & \left.\equiv N_{\mathrm{CS}}\right|_{t \rightarrow-\infty}-\left.N_{\mathrm{CS}}\right|_{t \rightarrow+\infty} \\
& =\frac{g^{2}}{64 \pi^{2}} \int d^{4} x \varepsilon^{\mu \nu \rho \sigma} F_{\mu \nu}^{a} F_{\rho \sigma}^{a} .
\end{aligned}
$$

The topological number (3) is given by the difference the Chern-Simons charge $N_{\mathrm{CS}}=N_{\mathrm{CS}}(t)$ at initial and final gluonic configurations.

After performing a chiral rotation of the quark fields, the topological term (2) disappears, and the $\theta$ dependence is transmitted from the gluonic topological term (2) to the quark sector in the form of the chiral (axial) chemical potential $\mu_{5}=\partial \theta / \partial t$. In our work, we use the NJL model to describe the dynamics of quarks. The gluon sector will thus leave its imprint only in the phenomenological interactions between quarks and in the mentioned chiral chemical potential.

The Lagrangian density of the quark model that we use in this study is given by the sum of the four terms,

$$
\mathcal{L}=\mathcal{L}_{q}+\mathcal{L}_{m}+\mathcal{L}_{4}
$$

where

$$
\mathcal{L}_{q}=\bar{\psi}\left(i \gamma^{\mu} \partial_{\mu}+\mu_{5} \gamma^{0} \gamma^{5}\right) \psi \equiv \bar{\psi} i \gamma^{\mu} \partial_{\mu} \psi+\mu_{5} n_{5}
$$

denotes the free quark contribution with the chiral chemical potential $\mu_{5}$ and the term

$$
\mathcal{L}_{m}=-m_{0} \bar{\Psi} \Psi
$$

introduces the current quark mass $m_{0}$ via the quark field dressed by interactions:

$$
\Psi(x)=\int d^{4} y G(x-y) \psi(y) .
$$

We need to introduce this special form of the mass term, Eqs. (6) and (7), instead of the standard term $\mathcal{L}_{m}^{(0)}=$ $-m_{0} \bar{\psi} \psi$, because we aim to model the perturbative tail of the current quark mass computed in the perturbative QCD at large Euclidean momentum. The perturbative matching will appear in the Fourier transform of the form factor $G(z)$ in Eq. (7) at large momentum $p$.

The last term in the quark Lagrangian (4) is a nonlocal interaction term that mimics the gluon-exchange effects,

$$
\begin{aligned}
\mathcal{L}_{4}= & G_{1} \sum_{\ell=0}^{3}\left[\left(\bar{Q} \tau_{\ell} Q\right)^{2}+\left(\bar{Q} i \gamma^{5} \tau_{\ell} Q\right)^{2}\right] \\
& +8 G_{2}\left[e^{i \theta} \operatorname{det}\left(\bar{Q}_{R} Q_{L}\right)+e^{-i \theta} \operatorname{det}\left(\bar{Q}_{L} Q_{R}\right)\right],
\end{aligned}
$$

where $\tau_{l}=(\mathbb{1}, i \boldsymbol{\tau})$ is a quaternion and the spinor $Q$ represents yet another dressed (nonlocal) quark field,

$$
Q(x)=\int d^{4} y F(x-y) \psi(y),
$$

expressed via the form factor $F(x-y)$ to be specified later. This form factor is similar to the function $G(x-y)$ that appears in the other form of the dressed quark field (7) used in the mass term of the quark Lagrangian (6). This interaction has been considered in its local version in Ref. [55] (see also references therein); therefore, we remind the reader of that study for further details.

It is convenient to perform the chiral rotation of the quark fields,

$$
\psi_{R} \rightarrow e^{-i \theta / 4} \psi_{R}, \quad \psi_{L} \rightarrow e^{i \theta / 4} \psi_{L},
$$

which removes the $\theta$ dependence in the interaction Lagrangian (8):

$$
\begin{aligned}
\mathcal{L}_{4}= & G_{1} \sum_{\ell=0}^{3}\left[\left(\bar{Q} \tau_{\ell} Q\right)^{2}+\left(\bar{Q} i \gamma^{5} \tau_{\ell} Q\right)^{2}\right] \\
& +8 G_{2}\left[\operatorname{det}\left(\bar{Q}_{R} Q_{L}\right)+\operatorname{det}\left(\bar{Q}_{L} Q_{R}\right)\right] .
\end{aligned}
$$

The $\theta$ dependence reappears in the quadratic part of the Lagrangian (4) in terms of the new fermionic fields, ${ }^{1}$

$$
\begin{aligned}
\mathcal{L}_{q}+\mathcal{L}_{m}= & \bar{\psi}\left(i \gamma^{\mu} \partial_{\mu}+\mu_{5} \gamma^{0} \gamma^{5}\right) \psi \\
& -\bar{\Psi}\left(m_{0+}+i m_{0-} \gamma^{5}\right) \Psi,
\end{aligned}
$$

via the rotated current masses:

$$
\begin{gathered}
m_{0+}=m_{0} \cos (\theta / 2), \\
m_{0-}=m_{0} \sin (\theta / 2) .
\end{gathered}
$$

It is also convenient to introduce the collective fields

$$
\begin{gathered}
\sigma=G_{+} \bar{Q} Q, \\
\eta=G_{-} \bar{Q} i \gamma^{5} Q ;
\end{gathered}
$$

we have put $G_{ \pm}=G_{1} \pm G_{2}$. Following the established procedure of bosonization at the one-loop approximation, we get the thermodynamic potential,

\footnotetext{
${ }^{1}$ For the notational convenience, we use the same symbols for the old and new fields, since the old ones will not appear again.
} 


$$
\begin{aligned}
\Omega= & \frac{\sigma^{2}}{G_{+}}+\frac{\eta^{2}}{G_{-}} \\
& -N_{c} N_{f} T \sum_{n} \int \frac{d^{3} p}{(2 \pi)^{3}} \log \beta^{4}\left(\omega_{n}^{2}+E_{+}^{2}\right)\left(\omega_{n}^{2}+E_{-}^{2}\right),
\end{aligned}
$$

where $\beta=1 / T$ is the inverse temperature and $\omega_{n}=$ $\pi T(2 n+1)$ with $n \in \mathbb{Z}$ are the fermionic Matsubara frequencies. In Eq. (17), we have also defined the energy branches,

$$
E_{ \pm}^{2}(p)=\left(p \pm \mu_{5}\right)^{2}+\mathcal{M}^{2}(p)+\mathcal{N}^{2}(p)
$$

with $p \equiv|\boldsymbol{p}|$ and

$$
\begin{aligned}
& \mathcal{M}(p)=m_{0+} \mathcal{R}(p)-2 \mathcal{C}(p) \sigma \\
& \mathcal{N}(p)=m_{0-} \mathcal{R}(p)-2 \mathcal{C}(p) \eta .
\end{aligned}
$$

Here, the function $\mathcal{C}(p) \equiv F^{2}(p)$ is determined via the Fourier transform $F(p)$ of the form factor in Eq. (9). The function $\mathcal{R}(p)=G(p)^{2}$ gives the evolution of the (renormalized) current quark mass with the UV scale that is necessary to reproduce the independence of the combination $m\langle\bar{q} q\rangle$ from the renormalization point.

The expectation (i.e., the mean field) values of the condensates $\sigma$ and $\eta$ are determined at each temperature $T$ and chiral chemical potential $\mu_{5}$ by the minimization of the thermodynamic potential $\Omega$. For sake of notational convenience, we have used $\sigma$ and $\eta$ to denote the mean field values of these fields. To derive Eq. (17), we used the imaginary time formalism to deal with the finite temperature bath and employed the analytical continuation to Euclidean momentum $p_{E}=\left(\boldsymbol{p}, p_{4}=-i p_{0}\right)$.

For future reference, it is useful to define the quantities

$$
\begin{gathered}
M\left(p_{E}\right)=m_{0} \mathcal{R}\left(p_{E}\right)-2 \sigma \mathcal{C}\left(p_{E}\right), \\
m\left(p_{E}\right)=m_{0} \mathcal{R}\left(p_{E}\right),
\end{gathered}
$$

which correspond to the quark mass function and the current mass at $\theta=0$, respectively.

We now specify the analytical forms of $\mathcal{R}(p)$ and $\mathcal{C}(p)$. For the latter, we follow Ref. [35] and take

$$
\begin{aligned}
\mathcal{C}\left(p_{E}\right)= & \theta\left(\Lambda^{2}-p_{E}^{2}\right) \\
& +\theta\left(p_{E}^{2}-\Lambda^{2}\right) \frac{\Lambda^{2}}{p_{E}^{2}} \frac{\left(\log \Lambda^{2} / \Lambda_{\mathrm{QCD}}^{2}\right)^{\gamma}}{\left(\log p_{E}^{2} / \Lambda_{\mathrm{QCD}}^{2}\right)^{\gamma}}
\end{aligned}
$$

here, $p_{E}$ is the Euclidean 4-momentum, and $\gamma=1-d_{m}$ is given by the anomalous dimension of the current quark mass for a two-flavor QCD:

$$
d_{m}=12 / 29
$$

The second line mimics the quark mass function computed in perturbative QCD arising from the chiral condensate $[33,34,56]$, while $\Lambda$ is an additional parameter of the model that corresponds to the momentum scale at which the perturbative mass is matched to the nonperturbative one. Differently from previous works, we have also introduced an energy scale dependence of the current mass, which mimics the running of this quantity computed in perturbative QCD [33,34,56]:

$$
\mathcal{R}\left(p_{E}\right)=\theta\left(\Lambda^{2}-p_{E}^{2}\right)+\theta\left(p_{E}^{2}-\Lambda^{2}\right) \frac{\left(\log \Lambda^{2} / \Lambda_{\mathrm{QCD}}^{2}\right)^{d_{m}}}{\left(\log p_{E}^{2} / \Lambda_{\mathrm{QCD}}^{2}\right)^{d_{m}}}
$$

The perturbative $p_{E}$ tails in the functions $\mathcal{C}\left(p_{E}\right)$ and $\mathcal{R}\left(p_{E}\right)$ ensure that the divergence of the chiral condensate with an UV cutoff $\Lambda_{\mathrm{UV}} \gg \Lambda$ is absorbed by that of the current mass so that the combination $m_{0}\langle\bar{q} q\rangle$ is independent from the UV cutoff $\Lambda_{\mathrm{UV}}$. Moreover, the log tail of the current quark mass will make the divergence of $n_{5}$ softer than that of a fermion gas with a momentum independent mass, as we discuss in Sec. III.

Strictly speaking, the thermodynamic potential (17) is a UV divergent quantity which has to be regularized at a proper subtraction point. In this study, we chose to subtract the potential with the vanishing condensates $\sigma=\eta=0$ at $T=\mu_{5}=0$ and $\theta \neq 0$, namely, at the free vacuum Fermi gas contribution at a finite topological angle $\theta$. This procedure is feasible since the subtracted quantity does not depend on the quark condensate, temperature, and $\mu_{5}$, and therefore it does not modify the thermodynamics of the system. Therefore, we work with the following form of the UV-regularized thermodynamic potential:

$$
\begin{aligned}
\Omega= & \frac{\sigma^{2}}{G_{+}}+\frac{\eta^{2}}{G_{-}} \\
& -N_{c} N_{f} T \sum_{n} \int \frac{d^{3} p}{(2 \pi)^{3}} \log \beta^{4}\left(\omega_{n}^{2}+E_{+}^{2}\right)\left(\omega_{n}^{2}+E_{-}^{2}\right) \\
& +2 N_{c} N_{f} \int \frac{d^{4} p_{E}}{(2 \pi)^{4}} \log \left(\frac{p_{E}^{2}+m_{0+}^{2}}{\Lambda^{2}}\right)
\end{aligned}
$$

We added the factor $\Lambda$ in the denominator of the last log function for the sake of the dimensional consistency of the equation. This addition is irrelevant for the computation of any physical quantity.

\section{B. Chiral condensate}

Before proceeding with the actual calculations, we discuss subtleties of the definition of the chiral condensate. We first focus on the $C P$-invariant case $\mu_{5}=0$; then, we generalize the discussion to the case of the chiral medium 
with $\mu_{5} \neq 0$. Also, we ignore a possible presence of the $\eta$ condensate (16) because this condensate breaks the time reversal symmetry $(T: t \rightarrow-t)$, while the chiral chemical potential is a $T$-even quantity.

In general, we can write for each quark flavor $q$

$$
\langle\bar{q} q\rangle=-\operatorname{Tr}\left(S-S_{0}\right),
$$

where $S$ corresponds to the full quark propagator and $S_{0}$ denotes the propagator of quarks with only the current mass taken into account. The subtraction in Eq. (27) is necessary to take into account only the contribution to $\langle\bar{q} q\rangle$ that comes from the interaction and not from the current quark mass.

It is well known that the chiral condensate diverges logarithmically with the renormalization scale in perturbative QCD [33,34]; this behavior is respected in our model. As a matter of fact, we have

$$
\begin{aligned}
\langle\bar{q} q\rangle= & -N_{c} \int \frac{d^{4} p_{E}}{(2 \pi)^{4}} \frac{4 M\left(p_{E}\right)}{p_{4}^{2}+\boldsymbol{p}^{2}+M\left(p_{E}\right)^{2}} \\
& +N_{c} \int \frac{d^{4} p_{E}}{(2 \pi)^{4}} \frac{4 m\left(p_{E}\right)}{p_{4}^{2}+\boldsymbol{p}^{2}+m\left(p_{E}\right)^{2}} .
\end{aligned}
$$

In the UV regime, each component of the Euclidean momentum $p_{E}$ should be taken much larger than the masses $M$ and $m$,

$$
\langle\bar{q} q\rangle_{\mathrm{UV}} \approx-4 \int_{\Gamma}^{\Lambda_{\mathrm{UV}}} \frac{d^{4} p_{E}}{(2 \pi)^{4}} \frac{\left[M\left(p_{E}\right)-m\left(p_{E}\right)\right]}{p_{E}^{2}},
$$

with $\Gamma>\Lambda$. From the above equation, it is clear that the subtraction in Eq. (27) leaves the contribution of the interaction as the only one that is taken into account for the evaluation of the chiral condensate. Moreover, a simple power counting shows that the integral possesses a log divergence in the limit $\Lambda_{\mathrm{UV}} \gg \Gamma$ due to the perturbative tail of $M\left(p_{E}\right)$.

The previous discussion can be generalized to a nonzero chiral chemical potential, $\mu_{5} \neq 0$. Instead of Eq. (28), we now have

$$
\begin{aligned}
\langle\bar{q} q\rangle= & -N_{c} \int \frac{d^{4} p_{E}}{(2 \pi)^{4}} \frac{4 M\left(p_{E}\right)\left[M\left(p_{E}\right)^{2}+p_{4}^{2}+\boldsymbol{p}^{2}+\mu_{5}^{2}\right]}{\left[p_{4}^{2}+\lambda_{+}^{2}\left(p_{E}\right)\right]\left[p_{4}^{2}+\lambda_{-}^{2}\left(p_{E}\right)\right]} \\
& +N_{c} \int \frac{d^{4} p_{E}}{(2 \pi)^{4}} \frac{4 m\left(p_{E}\right)\left[m\left(p_{E}\right)^{2}+p_{4}^{2}+\boldsymbol{p}^{2}+\mu_{5}^{2}\right]}{\left[p_{4}^{2}+\phi_{+}^{2}\left(p_{E}\right)\right]\left[p_{4}^{2}+\phi_{-}^{2}\left(p_{E}\right)\right]}
\end{aligned}
$$

where

$$
\begin{aligned}
& \lambda_{ \pm}^{2}\left(p_{E}\right)=\left(|\boldsymbol{p}| \pm \mu_{5}\right)^{2}+M\left(p_{E}\right)^{2} \\
& \phi_{ \pm}^{2}\left(p_{E}\right)=\left(|\boldsymbol{p}| \pm \mu_{5}\right)^{2}+m\left(p_{E}\right)^{2}
\end{aligned}
$$

Again in the ultraviolet regime in which each component of the Euclidean momentum $p_{E}$ is taken much larger than the masses $M$ and $m$. At the lowest order in $\mu_{5}$, we get

$$
\langle\bar{q} q\rangle_{\mathrm{UV}} \approx-4 \int_{\Gamma}^{\Lambda_{\mathrm{UV}}} \frac{d^{4} p_{E}}{(2 \pi)^{4}} \frac{\left[M\left(p_{E}\right)-m\left(p_{E}\right)\right]}{p_{E}^{4}}\left(p_{E}^{2}+\mu_{5}^{2}\right),
$$

with the infrared cutoff $\Gamma>\Lambda$. Using the power counting, we find that the chiral chemical potential gives a $\mu_{5}^{2}$ correction to the chiral condensate. This correction, proportional to $\left[M\left(p_{E}\right)-m\left(p_{E}\right)\right] / p_{E}^{4}$ is finite in the UV regime.

We finally remark that, although the chiral condensate has a UV log divergence, the condensate $\sigma=\langle\bar{Q} Q\rangle$ computed by minimization of the thermodynamic potential (26) is a finite quantity independent of the ultraviolet cutoff. Indeed, it is easy to realize that the gap equation, $\partial \Omega / \partial \sigma=0$, gives in this case

$$
\sigma=-2 N_{c} N_{f} T G_{+} \sum_{n} \int \frac{d^{3} p}{(2 \pi)^{3}} \frac{\mathcal{M}\left(\omega_{n}, p\right) \mathcal{C}\left(\omega_{n}, p\right)}{\omega_{n}^{2}+E_{+}^{2}},
$$

where we also used Eqs. (18) and (19). The loop integral on the right-hand side of the above equation is finite in the nonlocal NJL model due to the form factor (23) which removes high-momentum modes. Note that this factor does not appear in the chiral condensate. As a consequence, while in the local NJL model the quark condensate is proportional to $\sigma$, in the nonlocal model, this proportionality is lost [20].

\section{Chiral density}

\section{Thermodynamic definition}

In this subsection, we discuss the chiral density within the nonlocal NJL model. The chiral density

$$
n_{5}=-\frac{\partial \Omega}{\partial \mu_{5}}
$$

is given by the variation of the thermodynamic potential $\Omega$ with respect to the chiral chemical potential $\mu_{5}$. From Eq. (17), we get, at the minimum of $\Omega$,

$$
n_{5}=4 N_{c} N_{f} \mu_{5} \mathcal{H}
$$

with

$\mathcal{H}=T \sum_{n} \int \frac{d^{3} p}{(2 \pi)^{3}} \frac{\omega_{n}^{2}-\boldsymbol{p}^{2}+M\left(p_{E}\right)^{2}+\mu_{5}^{2}}{\left[\omega_{n}^{2}+\lambda_{+}^{2}\left(p_{E}\right)\right]\left[\omega_{n}^{2}+\lambda_{-}^{2}\left(p_{E}\right)\right]}$,

where the functions $\lambda_{ \pm}$are defined in Eq. (31) and the Euclidean momentum is determined at the Matsubara frequencies, $p_{E}=\left(\omega_{n}, \boldsymbol{p}\right)$. 


\section{Divergence of unrenormalized chiral density}

Before presenting the results on the chiral density $n_{5}$ obtained within the nonlocal NJL model, we find it useful to make a remark on the divergence of the density $n_{5}$ for a case when the quark mass is a fixed finite quantity. We limit this short discussion to the zero-temperature case, $T=0$, since the finite temperature part provides us with a finite contribution; in this case, we substitute $\omega_{n} \rightarrow p_{4}$ and take the integral over the continuous momentum $p_{4}$ along the full real axis.

If we set the dressed mass to zero, $M=0$, in Eq. (36), then the trivial integration over the momentum $p_{4}$ along the full real axis gives us the following expression for the zerotemperature chiral density:

$$
n_{5}=2 N_{c} N_{f} \int \frac{d^{3} p}{(2 \pi)^{3}} \theta\left(\mu_{5}-|\boldsymbol{p}|\right)=\frac{N_{c} N_{f} \mu_{5}^{3}}{3 \pi^{2}} .
$$

The above equation provides us with the standard relation between a density, $n$, and a chemical potential, $\mu$, of an ultrarelativistic massless fermion gas.

We now consider the effect of a momentum independent mass, $m_{0}$, on the chiral density $n_{5}$. From Eq. (36) after integrating over the momentum $p_{4}$ along the real axis, we get

$$
n_{5}=2 N_{c} N_{f} \int \frac{d^{3} p}{(2 \pi)^{3}} \mathcal{X}(p)
$$

where

$$
\begin{aligned}
\mathcal{X}\left(p, \mu_{5}\right)= & \frac{|\boldsymbol{p}|+\mu_{5}}{2 \sqrt{\left(|\boldsymbol{p}|+\mu_{5}\right)^{2}+m_{0}^{2}}} \\
& -\frac{|\boldsymbol{p}|-\mu_{5}}{2 \sqrt{\left(|\boldsymbol{p}|-\mu_{5}\right)^{2}+m_{0}^{2}}} .
\end{aligned}
$$

The above equation can be interpreted as a distribution function of a quark at $T=0, \mu_{5} \neq 0$, and $m_{0} \neq 0$. For a zero mass $m_{0}=0$, Eq. (40) naturally leads to Eq. (38), thus implying that a Fermi sphere is filled up to the Fermi momentum at $|\boldsymbol{p}|=\mu_{5}$.

The effect of the presence of the mass $m_{0}$ is to enlarge the chiral Fermi surface by putting particles above the Fermi momentum. Indeed, we may take in Eq. (40) the large spatial momentum $|\boldsymbol{p}|$ limit by assuming naturally the order $^{2} m_{0} \ll \mu_{5} \ll|\boldsymbol{p}|$ and obtain

$$
\mathcal{X}\left(p, \mu_{5}\right) \approx \frac{m_{0}^{2} \mu_{5}}{|\boldsymbol{p}|^{3}} .
$$

\footnotetext{
${ }^{2}$ Assuming this specific order for $m_{0}, \mu_{5}$, and $|\boldsymbol{p}|$ is irrelevant in the UV limit; this choice is closer to the nonlocal NJL model because of the running of the current quark mass in the UV.
}

Despite the distribution (41) decays as fast as $p^{-3}$, the density of the states increases proportional to the phasevolume factor $p^{2}$. Therefore, the net contribution to the chiral density behaves as $\int d p / p$, thus giving rise to a logarithmic divergence of the chiral density $n_{5}$ in the presence of a nonzero mass, $m_{0} \neq 0$.

The discussion of the example with $m_{0} \neq 0$ paves the way for understanding of the properties of the chiral density $n_{5}$ in the nonlocal NJL model. In the latter case, the integral over the momentum $p_{4}$ cannot be taken explicitly to the momentum dependence of the quark mass function $M=M\left(p_{E}\right)$. Nevertheless, we may figure out the UV divergence of the density $n_{5}$ because to this end it is enough to consider the asymptotic behavior of the integrand in Eq. (38) in the high-momentum limit $p_{4}, p \sim \Lambda_{\mathrm{UV}}$ with the large ultraviolet cutoff $\Lambda_{\mathrm{UV}} \gg \mu_{5}, M$.

We first expand the integrand in Eq. (37) in powers of the quark mass function $M$ at the lowest nontrivial order:

$$
\begin{aligned}
& \frac{p_{4}^{2}-\boldsymbol{p}^{2}+M\left(p_{E}\right)^{2}+\mu_{5}^{2}}{\left[p_{4}^{2}+\lambda_{+}^{2}\left(p_{E}\right)\right]\left[p_{4}^{2}+\lambda_{-}^{2}\left(p_{E}\right)\right]} \\
& \approx \frac{p_{4}^{2}-\boldsymbol{p}^{2}+\mu_{5}^{2}}{\left[p_{4}^{2}+\left(|\boldsymbol{p}|-\mu_{5}\right)^{2}\right]\left[p_{4}^{2}+\left(|\boldsymbol{p}|+\mu_{5}\right)^{2}\right]} \\
& +M^{2}\left(p_{E}\right) \frac{3 \boldsymbol{p}^{4}+2 \boldsymbol{p}^{2}\left(p_{4}^{2}-\mu_{5}^{2}\right)-\left(p_{4}^{2}+\mu_{5}^{2}\right)^{2}}{\left[p_{4}^{2}+\left(|\boldsymbol{p}|-\mu_{5}\right)^{2}\right]^{2}\left[p_{4}^{2}+\left(|\boldsymbol{p}|+\mu_{5}\right)^{2}\right]^{2}} \text {. }
\end{aligned}
$$

Since we are interested in the UV limit of this integrand, we can safely assume the hierarchy $M\left(p_{E}\right) \ll \mu_{5}$, which is valid due to the diminishing perturbative tail of the quark mass function $M\left(p_{E}\right)$.

The first term on the right-hand side of Eq. (42) leads to a finite integral and gives back the result (38) for the massless quarks while the UV divergence of the chiral density $n_{5}$ comes from the integral of the second term in the right-hand side of Eq. (42). The quark mass function $M\left(p_{E}\right)$ gets contributions from both the chiral condensate and the current quark mass (19). However, for a large Euclidean momentum $p_{E}$, the latter factor dominates since the former is suppressed by the perturbative tail $1 / p_{E}^{2}$ according to Eqs. (23) and (25).

Therefore, in the high momentum limit of Eq. (42), we can replace the quark mass function $M\left(p_{E}\right)$ with the mass $m\left(p_{E}\right)$. Moreover, in the UV region $p_{4},|\boldsymbol{p}| \gg \mu_{5}$, we can make an asymptotic expansion in powers of $\mu_{5} / p_{4}, \mu_{5} /|\boldsymbol{p}|$, to extract the behavior of the integrand at large Euclidean momentum, and at the lowest order, this amounts to setting $\mu_{5}=0$ in the second term in the right-hand side of Eq. (42) since higher powers of chiral chemical potential $\mu_{5}$ in a $\mu_{5} /\left|p_{E}\right|$ expansion would only lead to convergent integrals. Thus, we may finally rewrite Eq. (42) in the following form: 


$$
\begin{aligned}
& \frac{p_{4}^{2}-\boldsymbol{p}^{2}+M\left(p_{E}\right)^{2}+\mu_{5}^{2}}{\left[p_{4}^{2}+\lambda_{+}^{2}\left(p_{E}\right)\right]\left[p_{4}^{2}+\lambda_{-}^{2}\left(p_{E}\right)\right]} \\
& \approx \\
& \quad \frac{p_{4}^{2}-\boldsymbol{p}^{2}+\mu_{5}^{2}}{\left[p_{4}^{2}+\left(|\boldsymbol{p}|-\mu_{5}\right)^{2}\right]\left[p_{4}^{2}+\left(|\boldsymbol{p}|+\mu_{5}\right)^{2}\right]} \\
& \quad+m^{2}\left(p_{E}\right) \frac{3 \boldsymbol{p}^{2}-p_{4}^{2}}{p_{E}^{6}}+O\left(m^{2}\left(p_{E}\right) \mu_{5}^{2} / p_{E}^{6}\right) .
\end{aligned}
$$

It is now easy to recognize in the last term, proportional to the mass squared, $m^{2}\left(p_{E}\right)$, the source of the UV divergence of the chiral density $n_{5}$. This divergence would be of a $\log$ type if the mass $m$ were a constant quantity. The actual $p_{E^{-}}$ dependence of the mass $m=m\left(p_{E}\right)$ leads to somewhat smoother divergence. Indeed, taking into account the behavior of the aforementioned term at the momentum shell $\Gamma \ll\left|p_{E}\right| \ll \Lambda_{\mathrm{UV}}$, we get, ignoring an irrelevant proportionality constant,

$$
n_{5}^{\text {divergent }} \sim \mu_{5} m_{0}^{2}\left(\log \frac{\Lambda_{\mathrm{UV}}^{2}}{\Gamma^{2}}\right)^{1-2 d_{m}},
$$

where $d_{m}$ is the anomalous mass dimension (24). In a twoflavor QCD, the power of the logarithm in Eq. (44) is a small, but positive number: $1-2 d_{m}=5 / 29 \approx 0.17$.

\section{Zero-point origin of the divergence}

The divergence (44) of the chiral density $n_{5}$ occurs if and only if the current quark mass is nonzero, $m_{0} \neq 0$. In this section, we demonstrate that this divergence has a "vacuum" origin rather than a thermodynamic one.

The presence of the chiral chemical potential $\mu_{5}$ modifies the functional behavior of the energy of the fermionic modes (18), thus affecting the contribution to the oftenneglected part of the free energy which would normally be associated with the vacuum energy. Consider, for example, the simplest case of free fermions with the quark mass $m_{0}$. The positive-energy branch of the fermionic modes in the presence of the nonzero chiral potential $\mu_{5}$ has the form

$$
\varepsilon_{p}^{(\chi)}\left(\mu_{5}\right)=\sqrt{\left(|\boldsymbol{p}|-\chi \mu_{5}\right)^{2}+m_{0}^{2}},
$$

where $\chi= \pm 1$ labels the helicity of the mode.

As the chiral chemical potential modifies the spectrum of free field fluctuations, it should also modify the vacuum energy carried by these fluctuations. According to the standard rules of quantum field theory, this zero-point (ZP) contribution is given by the sum over all modes:

$$
\Omega_{\mathrm{ZP}}\left(\mu_{5}\right)=\sum_{\chi= \pm 1} \int \frac{d^{3} p}{(2 \pi)^{3}} \varepsilon_{p}^{(\chi)}\left(\mu_{5}\right) .
$$

In all other circumstances, this zero-point contribution is automatically neglected because it does not depend on physical parameters of the system such as temperature and chemical potential and, therefore, may be undoubtedly associated with the vacuum.

However in our case, the zero-point energy (46) depends on the chiral chemical potential $\mu_{5}$, and consequently, the free-energy term $\Omega_{\mathrm{ZP}}$ must be taken into account in addition to the conventional free energy of the system. The explicit dependence of the energy of the zero-point fluctuations (46) on the chiral chemical potential determines its nonzero contribution to the chiral charge density. In particular, the zero-point energy determines the chiral density at zero temperature, when the usual thermodynamic contribution vanishes,

$$
\begin{aligned}
\left.n_{5}\right|_{T=0} & =-\frac{\partial \Omega_{\mathrm{ZP}}}{\partial \mu_{5}} \\
& =\sum_{\chi= \pm 1} \int_{0}^{\infty} \frac{p^{2} d p}{\pi^{2}} \frac{\left(\mu_{5}-\chi|\boldsymbol{p}|\right)}{\sqrt{\left(\mu_{5}-\chi|\boldsymbol{p}|\right)^{2}+m_{0}^{2}}},
\end{aligned}
$$

which coincides with the functional form of Eqs. (39) and (40) obtained earlier in another way.

For strictly massless fermions, $m_{0}=0$, the expression under the integral (47) coincides with the hard Fermi cutoff, $\theta\left(\mu_{5}-|\boldsymbol{p}|\right)$, which usually appears in the thermodynamic (and not in the zero-point) part:

$$
\left.n_{5}\right|_{\substack{m_{0}=0 \\ T=0}}=\frac{1}{\pi^{2}} \int_{0}^{\infty} p^{2} d p \theta\left(\mu_{5}-|\boldsymbol{p}|\right)=\frac{\mu_{5}^{3}}{3 \pi^{2}} .
$$

We automatically recover the finite thermodynamic expression (38) for $N_{f}=N_{c}=1$.

As we already figured out, the behavior of the chiral density (47) changes qualitatively for massive fermions with $m_{0} \neq 0$. At a large momentum $|\boldsymbol{p}| \gg m,\left|\mu_{5}\right|$, the integral in Eq. (47) behaves as

$n_{5}(T=0)=\frac{\mu_{5} m_{0}^{2}}{\pi^{2}} \int^{\Lambda_{\mathrm{UV}}} \frac{d p}{p} \sim \frac{\mu_{5} m_{0}^{2}}{\pi^{2}} \log \frac{\Lambda_{\mathrm{UV}}}{m_{0}}+\ldots$

which leads precisely to the logarithmic divergence found already in Eq. (44) (with vanishing anomalous dimension, $d_{m}=0$, as it is appropriate to a free theory).

Evidently, this zero-point divergence of the chiral density (49) cannot be renormalized by a conventional subtraction method that is usually used with respect to the vacuum contribution. Moreover, the divergence of the chiral density has deeper roots since the zero-point contribution to the free energy (46) contains the logarithmically divergent part which depends on the chiral chemical potential as well. This divergence, however, does not affect the mass gap equation which may be regularized without requiring the renormalization of the chiral chemical potential [41]. Thus, in order to deal with a finite chiral chemical potential in the theories with a finite fermion mass, we need to modify the 
normalization prescription itself. We discuss this question in the next section.

Before finishing this section, we would like to make three comments.

First, this type of divergence does not occur in dense fermionic systems with vanishing chiral chemical potential. Indeed, the vector chemical potential $\mu$ shifts the particle energy modes linearly without modifying the functional form of the momentum dependence of the energy: $\sqrt{\left|\boldsymbol{p}^{2}\right|+m_{0}^{2}} \rightarrow \sqrt{\left|\boldsymbol{p}^{2}\right|+m_{0}^{2}} \pm \mu$. Therefore, the presence of the vector chemical potential does not affect the zeropoint energy.

Second, in a local NJL model, in which the constituent quark mass $M$ has no momentum dependence, we would get the constituent quark mass $M$ instead of the current quark mass $m_{0}$ in Eq. (44) which would give a divergence in the chiral limit as well. This is precisely the divergence that has been found in Ref. [39]. On the other hand, within the nonlocal NJL model, if we were in the chiral limit, we would have gotten a finite $n_{5}$. The divergence, as we have just demonstrated, appears due to the zero-point fluctuations which exist in both local and nonlocal models.

Third, the divergence of the chiral density $n_{5}$, in particular Eq. (44), are most probably valid in full QCD as well since both the fermionic spectrum (45) and the zeropoint energy (46) have a rather universal origin. One could counterargue that the present model represents a very crude description of the interaction that leads to the spontaneous chiral symmetry breaking of QCD. However, the model describes correctly the behavior of the quark mass function at large $p_{E}$, which is the region of interest for the discussion of the divergence of $n_{5}$, giving an argument that the result in Eq. (44) is applicable to QCD as well.

\section{Renormalization of the chiral chemical potential}

The chiral density has been computed in the firstprinciple numerical calculations in the scope of lattice QCD endowed with the dynamical rooted staggered fermions [42]. To this end, the Lagrangian has been shifted by a lattice version of the source term $\mu_{5} n_{5}$, which effectively induces a nonzero the chiral density $n_{5} \neq 0$ at a nonvanishing chiral chemical potential $\mu_{5} \neq 0$. While the UV divergence of the chiral density $n_{5}$ in the linear term has been also been noticed for naive formulation of lattice fermions, the full dynamical QCD calculations seem to support a finite result $n_{5} \sim \Lambda_{\mathrm{QCD}}^{2} \mu_{5}$, where $\Lambda_{\mathrm{QCD}}$ is a finite mass parameter of the order of a typical QCD energy scale. This result matches well the chiral perturbation theory $(\chi \mathrm{PT})$ which implies $n_{5} \sim f_{\pi}^{2} \mu_{5}$ for small $\mu_{5}$ at $T=0$ in the chiral limit [42]. Here, $f_{\pi} \sim \Lambda_{\mathrm{QCD}}$ is a pion decay constant. It is an easy exercise to prove that Eq. (36) is consistent with $\chi \mathrm{PT}$ at the lowest order in the chiral chemical potential $\mu_{5}$ and in the current quark mass by putting $\mu_{5}=0$ in the integrand and using Eq. (4.26) of Ref. [19].
We want to note, however, that we have been unable to reproduce the $\chi \mathrm{PT}$ relation $n_{5} \sim f_{\pi}^{2} \mu_{5}$ for a nonregularized chiral density $n_{5}$ in the context of the nonlocal NJL model. This relation is unlikely to hold in this model because the integrals for the naively-defined $n_{5}$ and the pion decay constant, $f_{\pi}$, entering at different sides of the relationship are pretty inconsistent (see, for example, Ref. [20]). In particular, $f_{\pi}$ is given in terms of convergent integrals, while the nonregularized $n_{5}$ still possesses a UV divergence in this model (44). In the nonlocal NJL model, we can renormalize the chiral condensate $n_{5}$ by removing the logarithmic divergence (44) similarly to a standard renormalization procedure in any well-defined renormalizable theory.

As we discussed in the previous section, the divergence appears from zero-point fluctuations of the fermionic modes which possess the unconventional energy dispersion in the presence of the chiral chemical potential. This feature appears both in the nonlocal NJL model (18) as well as in the case of free fermions (45). In the standard quantum field theory, the contribution from the zero-point fluctuations can easily be subtracted because it does not depend on the parameters of the matter sector of the theory. In our case, however, the zero-point term contains also a matter contribution which cannot be neglected. This leads to subtleties in the normalization procedure.

Postponing the physical justification of the formal renormalization procedure to a later discussion, we notice that the source of the zero-point ultraviolet divergence can be easily traced in Eq. (43). We define a renormalized chiral density as

$$
n_{5}^{\mathrm{R}}=4 N_{c} N_{f} \mu_{5}\left(\mathcal{H}-\mathcal{H}_{0}\right),
$$

where the counterterm

$$
\mathcal{H}_{0}=\int \frac{d^{4} p_{E}}{(2 \pi)^{4}} \frac{\left(3 \boldsymbol{p}^{2}-p_{4}^{2}\right) m\left(p_{E}\right)^{2}}{\left[p_{E}^{2}+m\left(p_{E}\right)^{2}\right]^{3}}
$$

is computed at zero temperature $T=0$ and it is independent of the chiral condensate.

According to the discussion in the previous section, the subtraction in the chiral density (51) is intimately connected to a similar renormalization of the zero-point contribution of the free energy (46). The renormalization of both these quantities does not explicitly involve the renormalization of the chiral chemical potential $\mu_{5}$. However, since $n_{5}$ and $\mu_{5}$ are thermodynamically conjugated quantities, the renormalization of the density at the leading order points to a possible renormalization of the associated chemical potential at the next-to-leading order.

When multiplied by the chiral chemical potential $\mu_{5}$ in Eq. (50), the regularized contribution depends only on $\mu_{5}$. The term $m\left(p_{E}\right)^{2}$ that appears in the denominator of the integrand in Eq. (51) makes it possible to avoid the 
apparent infrared divergence at low Euclidean momenta, $p_{E} \rightarrow 0$. This definition of the chiral density is consistent with Eq. (38) in the chiral limit, at which both the constituent and current masses are zero, $M=m=0$.

The subtraction in Eq. (50) is enough to cancel the mild logarithmic divergence of the chiral density (44) since higher-order terms in powers of the chemical potential $\mu_{5}$ in Eq. (43) would lead to convergent integrals. Moreover, we also verified that within the nonlocal NJL model at zero temperature $n_{5} \simeq C_{M} M_{q}^{2} \mu_{5}$, where $M_{q}$ denotes the quark mass function at $p=0$ and finite $\mu_{5}$. Numerically, the proportionality constant turns out to be $C_{M} \simeq 0.5$ at low chemical potentials up to $\mu_{5} \approx 300 \mathrm{MeV}$, while for larger chemical potentials $\mu_{5}$, the proportionality becomes softer, finally reaching $C_{M} \simeq 0.3$ at $\mu_{5}=400 \mathrm{MeV}$. These results are in agreement with the lattice QCD studies reported in Ref. [42] in which it is found that the chiral density $n_{5}$ at zero temperature is of the order of the anticipated value $\mu_{5} \Lambda_{\mathrm{QCD}}^{2}$.

The straightforwardly defined chiral density is finite in the chiral limit (where the fermion mass is zero) and divergent for the massive fermions (44). Therefore, it is natural to suggest that the divergence of the chiral density (44) is directed related to the presence of the fermions' mass and, naturally, to the inconsistency of the notion of the chiral chemical potential for fermions with a nonzero mass. Indeed, the chiral charge is not conserved as it dissolves via the chirality flips for a massive fermion regardless of the origin of it mass which could be either a current mass or a dynamically generated mass. For a free Dirac fermion with a mass $M$, the axial (chiral) current (1) has a nonzero 4-divergence at the level of the classical equations of motion:

$$
\partial_{\mu} j_{5}^{\mu}=2 i M \bar{\psi} \gamma^{5} \psi
$$

For strictly massless fermions, the chirality is a conserved number: $\partial_{\mu} j_{5}^{\mu}=0$.

A generic chemical potential has a well-defined meaning only for conserved quantities such as the electric (vector) charge. One may alternatively say that no chemical potential can thermodynamically be conjugated to a nonconserved quantity. In the photodynamics, for example, it is impossible to self-consistently introduce a chemical potential for the total number of photons as the latter number is evidently not conserved. In a free theory, such a "chemical potential," associated with a nonconserved quantity, decouples from the dynamics of the theory. In an interacting theory, this type of chemical potential may affect the dynamics as we discuss below.

In QCD, the chiral properties of fermions are connected to the topology of the gluonic sector of the theory. For example, the instanton- and sphaleron-induced transitions between adjacent topologically distinct vacua induce changes in the chiral charge of the fermions due to the axial anomaly in QCD,
$\left.\left(N_{R}-N_{L}\right)\right|_{t \rightarrow-\infty}-\left.\left(N_{R}-N_{L}\right)\right|_{t \rightarrow+\infty}=-2 N_{f} \Delta N_{\mathrm{CS}}$,

where $N_{5} \equiv V n_{5}=N_{R}-N_{L}$ is the difference between the numbers of fermions possessing the right-handed $\left(N_{R}\right)$ and left-handed $\left(N_{L}\right)$ chiralities, and change $\Delta N_{\mathrm{CS}}$ in the Chern-Simons number between initial and final configurations is given by the topological charge (3) of the gluonic configuration.

Since the fermion and gluon sectors of the theory are interacting with each other, a finite chiral chemical potential may also induce a response in the topological gluon sector in thermal equilibrium of QCD. Therefore, the finite chiral chemical potential may have a physical sense in QCD with massive fermions, regardless of the fact that the chiral number is not a conserved quantity. The relaxation of the chiral density may induce physical changes in the topological charge fluctuations in the gluonic sector of the theory. We will see below that the topological susceptibility of the theory is seemingly correlated with the chiral density in the background of the chiral chemical potential.

Since the chiral charge is not conserved (52), the introduction of the chiral chemical potential (5) cannot be justified in the thermodynamical sense. On the contrary, the chiral chemical potential $\mu_{5}$ should be treated as a new coupling of QCD, which-in general-needs a renormalization. The corresponding thermodynamically conjugated quantity, the density, should also require a renormalization as well (for example, in QCD, both the gluon coupling and gluon fields/strength tensors are renormalized perturbatively). Therefore, Eq. (50) defines nothing but a certain renormalization scheme in the chiral sector.

\section{RESULTS}

In this section, we report the main results of our study on the nonperturbative quantities associated with the chiral quark density in the scope of the nonlocal NJL model. Below, we discuss the dynamical quark mass $M$; the chiral condensate $\langle\bar{q} q\rangle$; the chiral density $n_{5}$; the topological susceptibility $\chi_{\text {top }}$; and, finally, the phase diagram in the $\left(\mu_{5}, T\right)$ parameter plane as originates from the nearly critical behavior of the chiral density and the topological susceptibility.

First, we fix the parameters of the NJL model. We take $\Lambda=550 \mathrm{MeV}$ for the matching scale and $m_{0}=5 \mathrm{MeV}$ for the undressed quark mass. Finally, the value of quartic quark interaction constant $G=2.6 / \Lambda^{2}$ and $G_{1}=$ $(1-c) G_{1}$ is chosen in order to reproduce the phenomenological value of the light quark condensate $\langle\bar{q} q\rangle=$ $(-250 \mathrm{MeV})^{3}$ in the vacuum at $T=\mu_{5}=0$, and $G_{2}=c G$ with $c=0.2$ [55]. In the numerical computations, we regularize the thermodynamic potential (17) by summing over all the Matsubara's frequencies and restricting the integration over the 3-momentum at the momentum sphere $|\boldsymbol{p}| \leq \Lambda_{\mathrm{UV}}$ with the ultraviolet cutoff $\Lambda_{\mathrm{UV}}=3 \Lambda$ (we have 
verified that changing this UV cut does not change the results drastically).

\section{A. Catalysis of chiral symmetry breaking}

Our first aim is demonstrate that the nonlocal NJL model is capable of describing the catalysis of chiral symmetry breaking induced by $\mu_{5}$, at least for relatively moderate values of the latter, $\left|\mu_{5}\right| \lesssim \Lambda$. To this end, we first discuss the constituent quark mass and the quark condensate at finite temperature $T$ and chiral chemical potential $\mu_{5}$. As some features of the quark mass at $\mu_{5} \neq 0$ were already been discussed in Refs. [35,36], here we limit ourselves to showing the evolution of the quark mass at zero Euclidean momentum $p_{E}=0$ with varying $T$ and $\mu_{5}$. For the computation of the quark mass, we restrict ourselves to vanishing topological angle $\theta=0$. Therefore, the pseudoscalar condensate vanishes, $\eta=0$, while the chiral condensate $\sigma$ is the only condensate left.

\section{Constituent quark mass}

In Fig. 1, we plot the constituent quark mass at zero Euclidean momentum versus temperature. The figure indicates that the increase in the chiral misbalance enhances the chiral symmetry breaking at any temperature in agreement with conclusions of Ref. [57].

In our model, we have verified that the catalysis of the chiral symmetry breaking occurs up to $\mu_{5} \approx \Lambda$. Above this value, the constituent mass decreases with $\mu_{5}$, but this feature might be related to the fact that we have not included any backreaction on the interaction kernel. We also notice that the constituent mass decreases in a narrow range of temperatures; there is a crossover to a hightemperature phase in which the chiral symmetry is

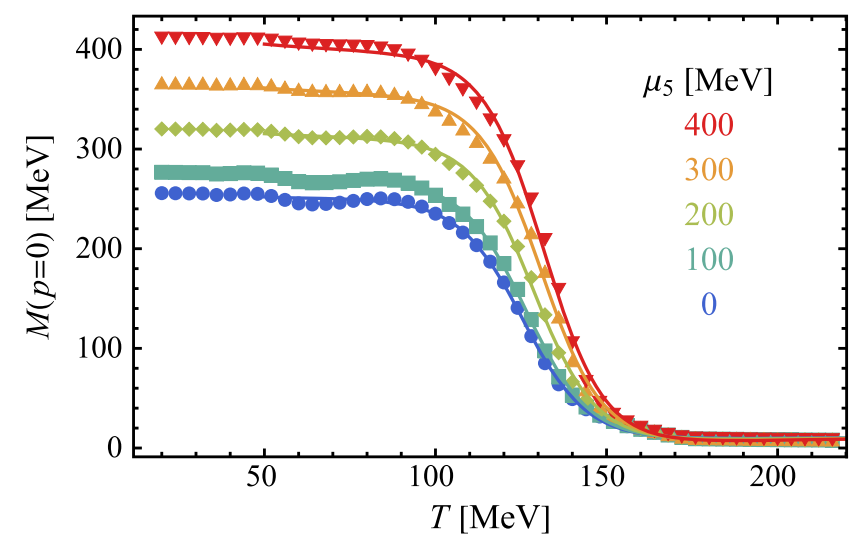

FIG. 1. Constituent quark masses $M$ at a zero momentum $p=0$ vs temperature $T$. The blue dots denote a vanishing chiral chemical potential $\mu_{5}=0$; the green squares stand for $\mu_{5}=100 \mathrm{MeV}$; the light green diamonds correspond to $\mu_{5}=200 \mathrm{MeV}$; the orange upward-pointing triangles stand for $\mu_{5}=300 \mathrm{MeV}$; and, finally, the red downward-pointing triangles denote $\mu_{5}=400 \mathrm{MeV}$. The solid lines represent the best fits (54). approximately restored. The smoothness of the transition appears to be supported by thermal fluctuations. We will see in the next section that the chiral symmetry restoration is accompanied by the approximate restoration of the $U(1)_{A}$ symmetry as well.

One way to determine position of the thermal crossover is to identify it, at a fixed chemical potential $\mu_{5}$, with the temperature $T_{c}=T_{c}\left(\mu_{5}\right)$ at which the absolute value of the slope of the infrared quark mass $\left|d M\left(p_{E}=0\right) / d T\right|$ takes its maximum. For example, one finds $T_{c} \simeq 125 \mathrm{MeV}$ at $\mu_{5}=0$, which agrees very well with the value quoted earlier in Ref. [18]. Figure 1 also implies that the critical temperature is an increasing function of $\mu_{5}$ in the whole range of chemical potentials $\mu_{5}$ covered by this study.

A more accurate way to find the thermal crossover is to find a suitable fitting function that may smoothly interpolate the low- and high-temperature behavior of the constituent quark mass. And indeed, this quantity may be well described in a wide region of temperatures by the function (with $\mathcal{O}=M$ in the considered case)

$\mathcal{O}\left(\mu_{5}, T\right)=C_{1}\left(\mu_{5}\right) T^{\nu} \tanh \left(\frac{T-T_{c}\left(\mu_{5}\right)}{\delta T_{c}\left(\mu_{5}\right)}\right)+C_{2}\left(\mu_{5}\right)$,

where $C_{1}, C_{2}, \nu, T_{c}$, and $\delta T_{c}$ are the fitting parameters defined at each fixed value of the chiral chemical potential $\mu_{5}$. The power $\nu$ is usually quite small ( $\nu \sim 0.1$ or smaller). Below, we will use the generic function (54) to describe other quantities in the pseudocritical region. We do not put the superscript $\mathcal{O}$ to the fitting parameters to keep our notations concise.

The best fits of the mass gap $M$ by the function (54) are shown in Fig. 1 by the solid lines. The pseudocritical temperature $T_{c}=T_{c}\left(\mu_{5}\right)$ and the width $\delta T_{c}=\delta T_{c}\left(\mu_{5}\right)$ the pseudocritical region, as determined by the mass gap $M \equiv M(p=0)$, follow very closely the corresponding quantities for the chiral condensate and the topological susceptibility, which we will discuss in more detail below.

\section{Chiral condensate}

The catalysis of the chiral symmetry breaking, induced by the chiral chemical potential $\mu_{5}$, is also evident from the behavior of the chiral condensate, $\langle\bar{q} q\rangle$, as defined in Eq. (27). In Fig. 2, we plot the chiral condensate for one of the light quarks, $\sigma_{u} \equiv\langle\bar{u} u\rangle$, versus temperature for several fixed values of $\mu_{5}$. The magnitude of the chiral condensate increases with the rise in the chiral chemical potential $\mu_{5}$. Thus, we have yet another confirmation that the chiral chemical potential acts as a catalyzer of the chiral symmetry breaking.

The approximate restoration of the chiral symmetry at a finite temperature is via a smooth crossover and not via a real thermodynamic phase transition. In the absence of a thermodynamic singularity in the parameter space, the very definition of the critical temperature leaves a large room for 


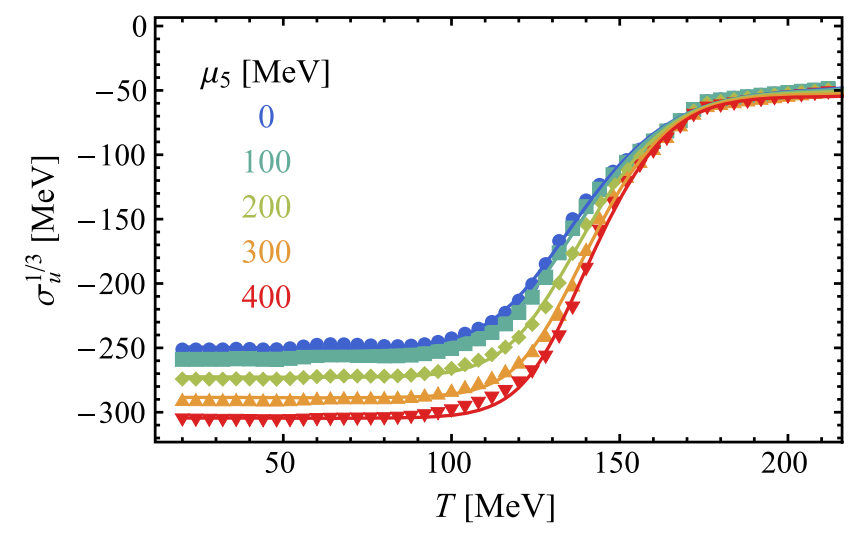

FIG. 2. Chiral condensate $\sigma_{u} \equiv\langle\bar{u} u\rangle$ vs temperature. Conventions for colors and symbols are the same as in Fig. 1. The solid lines represent the best fits (54).

ambiguities. Moreover, the transition temperature depends not only on the method of its definition but also on the particular thermodynamic quantity used to identify the temperature. The chiral condensate $\sigma_{u} \equiv\langle\bar{u} u\rangle$ (and, equivalently, for $\sigma_{d} \equiv \sigma_{u}$ ) may be described by the same type of function that has also been used for the constituent quark (54). The best fits are shown in Fig. 1 by the solid lines. We will discuss the pseudocritical temperature $T_{c}=T_{c}\left(\mu_{5}\right)$ and the width $\delta T_{c}=\delta T_{c}\left(\mu_{5}\right)$ in more detail at the end of this section.

\section{B. Chiral density}

In Fig. 3, we plot the regularized chiral density $n_{5}$, defined in Eqs. (50) and (51), versus temperature $T$ for several values of the chiral chemical potential $\mu_{5}$. The qualitative trend of $n_{5}$ versus temperature is obviously the same for every fixed $\mu_{5}$ : as temperature rises, the chiral density increases. This property is related to the fact that thermal excitations contribute more to the thermodynamic

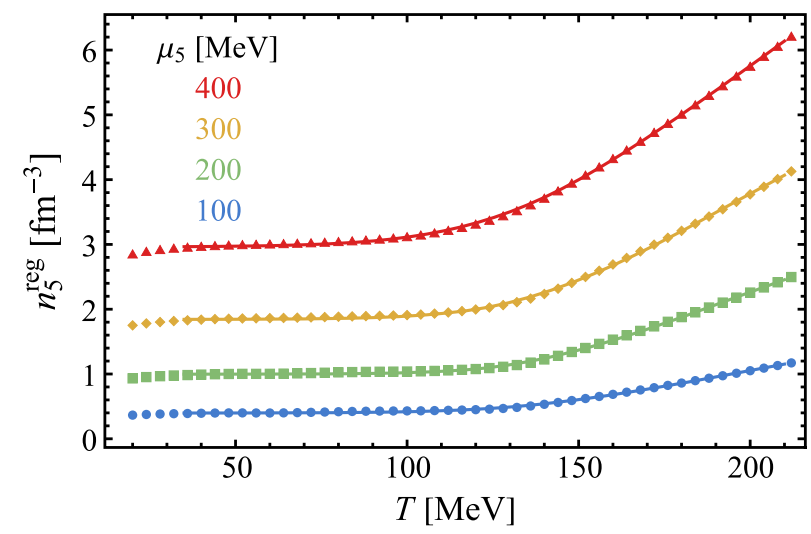

FIG. 3. Regularized chiral density $n_{5}$, defined via Eqs. (50) and (51), vs temperature. Conventions for colors and symbols are the same used in Fig. 1. The solid lines show the fits by the function (55). potential at temperature rises. Alternatively, the increase of the chemical potential $\mu_{5}$ at any fixed temperature $T$ results in the increase of the chiral density.

For every fixed chiral chemical potential $\mu_{5}$, the chiral density exhibits a kneelike structure which separates the low-temperature from high-temperature behavior, as seen in Fig. 3. This behavior may be described with a very good accuracy by the function

$$
n_{5}\left(\mu_{5}, T\right)=n_{1}\left(\mu_{5}\right) F\left(\frac{T-T_{0}\left(\mu_{5}\right)}{\delta T_{0}\left(\mu_{5}\right)}\right)+n_{2}\left(\mu_{5}\right),
$$

where $F(x)=\ln \left(1+e^{x}\right)$. The fitting parameters are $n_{1}$, $n_{2}, T_{0}$, and $\delta T_{0}$, where the temperature $T_{0}$ discriminates between the low- and high-temperature behavior, while the quantity $\delta T_{0}$ has a sense of the width of the transition region. All fitting parameters are the functions of the chiral chemical potential $\mu_{5}$. The best fits are shown in Fig. 3 by the solid lines.

Since the chiral density $n_{5}$ is not an order parameter of the deconfining transition, the knee temperature $T_{0}$ does not have a meaning of a (pseudo)critical temperature, especially, for a crossover transition. We get $T_{0} \simeq 135 \mathrm{MeV}$ with the width $\delta T_{0} \simeq 20 \mathrm{MeV}$.

\section{Topological susceptibility}

The topological susceptibility measures the strength of fluctuations of the topological charge in the medium. In our model, the topological susceptibility can be computed as the curvature:

$$
\chi_{\text {top }}=\left.\frac{\partial^{2} \Omega}{\partial \theta^{2}}\right|_{\theta=0} .
$$

It is well known that for two degenerate flavors of light quarks the topological susceptibility (56) is related to the quark condensate $|\langle\bar{q} q\rangle|$ as follows: $\chi_{\text {top }}=m|\langle\bar{q} q\rangle|$ where $m$ is the current quark mass. This relation, valid at zero temperature, shows the link between the fluctuations of the topological charge (56) and the dynamics of the light quark flavors that leads to the spontaneous chiral symmetry breaking. First-principle lattice simulations [58-60] indicate that the topological susceptibility tends to decrease with rising temperatures and that the axial symmetry tends to be restored at high temperatures at the chirally unbroken phase.

In a local NJL model, the topological susceptibility with light quarks in the chiral medium was studied for the first time in Ref. [39]. It has also been recently subjected to the first-principle lattice calculations at zero temperature in Ref. [42]. Both studies agree qualitatively with each other on the fact that at zero temperature $\chi_{\text {top }}$ increases with $\mu_{5}$, while a quantitative comparison is not feasible due to the different quark masses used in these calculations. 
On the other hand, the local NJL model [39] indicates that when the temperature is close to $T_{c}$ the chiral chemical potential tends to lower $\chi_{\text {top. }}$. This property is an artifact of the local interaction and of the three-dimensional regulator used in Ref. [39]. Already with a four-dimensional regulator, the critical temperature $T_{c}$ increases with the increase of the chiral chemical potential $\mu_{5}$, at least for small $\mu_{5}$ [35]. Cutoff effects become substantial at large values of $\mu_{5}$ in any regularization scheme, and $T_{c}$ tends to be lowered with the rise of $\mu_{5}$. For this reason, here we compute $\chi_{\text {top }}$ both at zero and at finite temperature by using the nonlocal NJL model, which offers a more trustable response of critical temperature $T_{c}$ considered as the function of the chiral chemical potential $\mu_{5}$.

In Fig. 4, we plot $\chi_{\text {top }}$ versus temperature. In the figure, the susceptibility is given in units of its vacuum value,

$$
\left.\chi_{0}^{1 / 4} \equiv \chi_{\mathrm{top}}^{1 / 4}\right|_{T=\mu_{5}=0} \simeq 76 \mathrm{MeV}
$$

where the numerical number is presented for the set of parameters used in our study. The value of the topological susceptibility (57) matches well the benchmark result of numerical simulations in full QCD [61], $\chi_{0}=$ 75.6(1.8)(0.9) MeV.

We notice that the increase the chiral density results in the increase of the topological susceptibility at each fixed temperature. This result is in a disagreement with previous calculations that use the local NJL model [39], which instead predict an increase of $\chi_{\text {top }}$ at small $T$ but a decrease of $\chi_{\text {top }}$ at large $T$. The result of Ref. [39] was obtained within a local NJL model; thus, the behavior of $\chi_{\text {top }}$ in that model follows that of the constituent quark mass at finite $\mu_{5}$.

Figure 4 indicates that there is a narrow range of temperatures in which the susceptibility $\chi_{\text {top }}$ decreases abruptly, thus signaling the partial restoration of the axial $U(1)_{A}$ symmetry. To extract the pseudocritical temperature

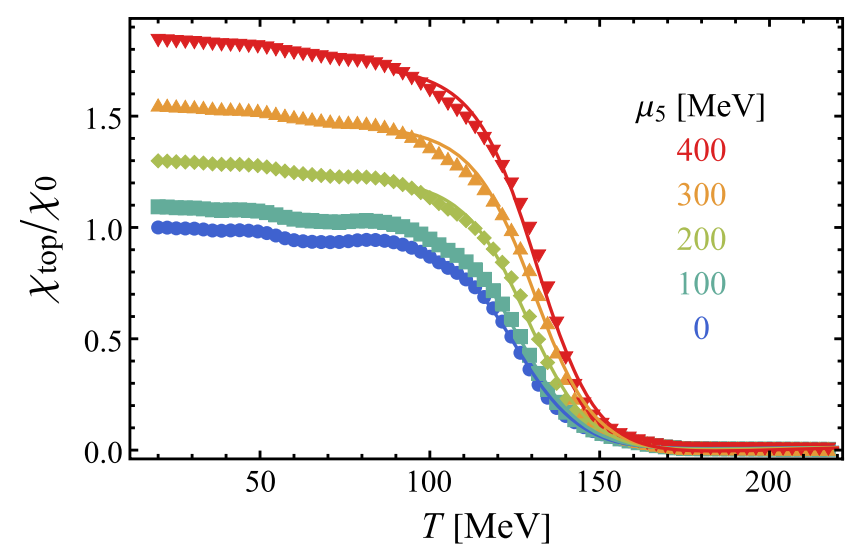

FIG. 4. Topological susceptibility (56) vs temperature, in units of the vacuum susceptibility $\chi_{0}(57)$. Conventions for colors and symbols are the same used in Fig. 1. The solid lines represent the best fits by the function (54). more accurately, we describe the topological susceptibility by the function (54). The best fits are shown in Fig. 4 by the solid lines. In the next subsection, we will discuss the pseudocritical temperature $T_{c}$ for this topological crossover in more detail.

\section{Pseudocritical temperatures}

In Fig. 5, we compare the pseudocritical temperatures $T_{c}$ of the chiral and axial crossovers, as well as their thermal widths, as functions of the chiral chemical potential $\mu_{5}$.

The figure shows a few interesting qualitative features of the chiral phase transition. First of all, both pseudocritical temperatures rise in unison as the chiral density increases. Second, the restoration of the axial symmetry, as revealed by the topological susceptibility, appears at a higher temperature than the restoration of the chiral symmetry. This statement is independent of the chiral chemical potential. At large values of the chiral chemical potential, we observe a flattening of the both pseudocritical temperatures, which, however, should be attributed to the crudeness of the model used in our studies. In particular, we have neglected any possible backreaction induced by the chemical potential $\mu_{5}$ on the form factor. From this point of view, the computation of the quark mass function at finite $\mu_{5}$ using the Schwinger-Dyson equation, which should allow one to incorporate the aforementioned dependence in the mass form factor, would be interesting.

The widths of both axial and chiral crossover transitions, $\delta T_{c}$, show a tendency for shrinking as the chiral matter gets denser, thus implying a strengthening of both transitions as the chiral chemical potential increases. However, we see no

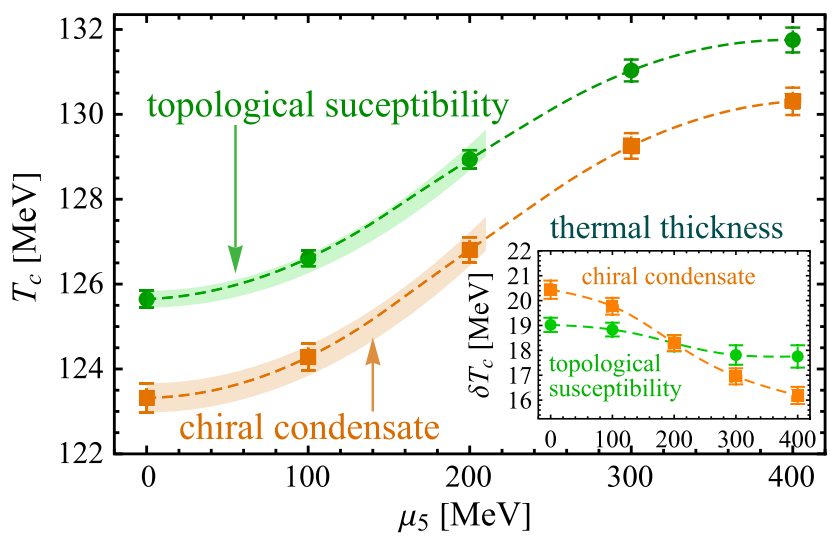

FIG. 5. Pseudocritical temperatures $T_{c}$ of the chiral symmetry restoration (the orange squares) and the axial symmetry restoration (the green circles) as functions of the chiral chemical potentials $\mu_{5}$. The inset shows the thickness of each transition. The data are obtained by fits of, respectively, the chiral condensate (Fig. 2) and the topological susceptibility (Fig. 4); see the text for more details. The dashed lines are drawn to guide the eye. The shaded regions represent the quadratic-curvature behavior of Eq. (58). 
signature of a critical end point in the $\left(\mu_{5}, T\right)$ phase diagram in the studied region of the chiral chemical potential $\mu_{5}$.

These conclusions are in certain disagreement with previous studies that used a local interaction kernel $[37,38]$ as well as with recent works using the Wigner function technique [62]. On the other hand, the absence of the phase transition point in the $\left(\mu_{5}, T\right)$ plane agrees well with the results obtained within the nonlocal NJL models $[35,36,63]$, with the solution of the Schwinger-Dyson equations [45-47], and with the first-principle QCD studies in a limited range of temperatures and chemical potentials [43].

A quantitative analysis reveals a less sharp picture given the smooth nature of both crossover transitions. First of all, the magnitude of the increase of both axial and chiral pseudocritical temperatures is very small; the rise of the chemical potential, from $\mu_{5}=0$ to a rather large value $\mu_{5}=400 \mathrm{MeV}$, leads to the enhancement of the critical temperature by about $\Delta T_{c} \simeq 5 \mathrm{MeV}$, or less than by $5 \%$. This variation of the temperature is located well within the broad widths of both crossovers, which are wider than $15 \mathrm{MeV}$ for all studied values of the chiral chemical potential. Moreover, at each given chiral density, both crossovers overlap strongly since the difference in the axial and chiral pseudocritical temperatures is within (2-3) MeV.

With both crossover transitions appearing so close to each other, we expect that the effects of the chiral- and axial-symmetry restorations will happen smoothly and simultaneously. As the temperature rises, (i) the meson masses will experience a small deep due to the diminishing mass gap at the chiral crossover, followed by (ii) a rise in the masses due to the thermal effects. The gradual restoration of the axial symmetry will have its imprint in the particle spectrum via enhancing the degeneracy of the whole nonet of the pseudoscalar bosons as the masses of all axial partners will approach each other. We refer the reader to Ref. [64] for a recent detailed discussion.

We also determine the curvature $\kappa_{5}$ of the crossover transition in the $\left(\mu_{5}, T\right)$ plane,

$$
\frac{T_{c}\left(\mu_{5}\right)}{T_{c}(0)}=1-\kappa_{5} \frac{\mu_{5}^{2}}{T_{c}^{2}(0)}+\ldots
$$

which is usually applied to the low-density domain with $\left|\mu_{5}\right| \ll T_{c}$. According to the conventions used in the literature, a positive curvature $\kappa$ corresponds to a diminishing (pseudo)critical temperature as the chemical potential increases. In a realistic QCD with three quark flavors $\left(N_{f}=2+1\right.$, with two light $u$ and $d$ quarks and one heavier $s$ quark), the baryonic curvature $\kappa_{B}$ determines the curvature of the pseudocritical temperature with respect to increase of the baryonic potential $\mu_{B}=3 \mu$ (see, for example, the recent studies in Ref. [65]),

$$
\frac{T_{c}\left(\mu_{5}\right)}{T_{c}(0)}=1-\kappa_{B} \frac{\mu_{B}^{2}}{T_{c}^{2}(0)}+\ldots
$$

where $\mu \equiv \mu_{q}$ is the quark chemical potential. In our article, we identify the chiral curvature (58) with respect to the chiral (axial) chemical potential $\mu_{5} \equiv \mu_{A}$.

The curvatures of the chiral and axial crossovers for the chiral quark chemical potential approximately coincide and give $\kappa_{5}^{\text {axial }}=-0.0105(4)$ obtained from the topological susceptibility and $\kappa_{5}^{\text {chiral }}=-0.0108(3)$ as extracted from the chiral condensate. The corresponding quadratic dependencies are shown in Fig. 5 by the shaded regions. The width of each region corresponds to the statistical error. We would like to notice that the quadratic dependence of the critical temperature on the chiral chemical potential holds very well for the relatively large values of the chiral chemical potential, $\mu_{5} \sim T_{c}$.

\section{CONCLUSIONS}

We have reported on our study of the chiral and axial symmetry breaking in chirally imbalanced QCD with two flavors of light fermions at finite temperature using a nonlocal Nambu-Jona-Lasinio model. We studied the chiral condensate, $\langle\bar{q} q\rangle$; the topological susceptibility, $\chi_{\text {top }}$; and chiral density, $n_{5}$, of a chiral medium, namely, a system with chiral chemical potential $\mu_{5} \neq 0$, at finite temperature. All the calculations have been performed within a nonlocal NJL model with quark mass function that agrees with perturbative QCD at large Euclidean momentum. Our approach differs from almost all of the previous calculations in which local effective models have been used. The use of a nonlocal NJL model is favored over the local ones since the former predicts that the critical temperature for the approximate chiral symmetry restoration, $T_{c}$, increases with $\mu_{5}$ in agreement with the solution of the Schwinger-Dyson equations as well as with the firstprinciple lattice QCD calculations. On the contrary, the conclusions of our approach disagree with the predictions of most of the local the NJL models (see Ref. [41] for an exception) as well as with the results obtained recently within the Wigner function approach.

The response of the chiral condensate to a finite chiral charge density at zero and finite temperature shows that the chiral chemical potential $\mu_{5}$ serves as a catalyzer of chiral symmetry breaking: the chiral condensate strengthens as the chiral density increases. This conclusion is in agreement with other studies [57]. Moreover, the behavior of the topological susceptibility at a finite chiral chemical potential indicates that the chiral medium tends to break the axial symmetry as well; the topological susceptibility becomes larger with increase of the chiral density at all studied temperatures. In other words, the chiral medium increases the fluctuations of the topological charge, thus enhancing the breaking of the axial $U(1)_{A}$ symmetry. We have 
confirmed that the critical temperature of the chiral crossover rises with $\mu_{5}$; we also noted the same behavior for the axial crossover and pointed out an apparent hierarchy of the temperatures $T_{c}^{\text {axial }}>T_{c}^{\text {chiral }}$. However, the axial and chiral crossovers possess substantial thermal widths, $\delta T_{c} \sim(15-20) \mathrm{MeV}$, which imply that these transitions overlap as $T_{c}^{\text {axial }}-T_{c}^{\text {chiral }} \sim(2-3) \mathrm{MeV}$. Thus, in our model, the axial symmetry restoration happens simultaneously with chiral symmetry restoration.

Part of this study has been devoted to the divergence of the chiral density. We argued that the presence of the chiral chemical potential should be treated as a Lorentz-framedependent coupling. The main argument is that the quarks get substantial masses due the chiral symmetry breaking, while the chiral charge is not a classically conserved quantity for the massive fermions. Therefore, the corresponding thermodynamically conjugated chiral chemical potential should not, therefore, be considered as a true chemical potential. The divergence of an unrenormalized chiral density (44) is a consequence of this property.

Technically, the presence of a nonzero chiral chemical potential modifies the functional form of the momentum dependence of the fermionic eigenenergies. The latter contributes to the zero-point energy which is no longer associated with the pure vacuum contribution due to the apparent dependence of the chiral chemical potential. Consequently, the zero-point fermionic fluctuations contribute to the density of the chiral charge. The zero-point contribution is finite for massless fermions, but it gives a logarithmically divergent term if the fermions have a mass. This fact highlights thermodynamic incompatibility between formation of a finite chiral density and the absence of the chiral symmetry for massive fermions. In the response, the system generates an ultraviolet divergent contribution to the free energy $\Omega \sim m_{0}^{2} \mu_{5}^{2} \ln \Lambda / m_{0}$, which forces the dynamical system to make the chiral chemical potential $\mu_{5}$ vanishing. In our work, we suggest that this divergence may, however, be regularized and then renormalized in order to describe transient phenomena with a nonzero chiral density in the theories with dynamical mass generation (for example, in quark-gluon plasma formed in heavy-ion collisions).

To support the need of the renormalization of the chiral chemical potential in QCD with nearly massless quarks, we invoked the following chain of arguments: the bare chemical potential creates a chiral charge density, that tends to decay due to chirality flips that are catalyzed by the presence of the dynamical mass; the dynamical mass appears as a result of the interactions of the theory, and the interactions require the renormalization of the corresponding couplings and observables. Thus, the processes that involve the (non)conservation of the chiral density are affected by the flow in the renormalization-group space of QCD, so the chiral charge and the thermodynamically conjugated chiral chemical potential should also be affected by the renormalization. This statement also applies to the theories where the quark mass appears dynamically as a result of the spontaneous breaking of chiral symmetry.

It will be interesting to check whether these predictions are valid also in a model with three dynamical flavors and to explore the behavior of different topological susceptibilities to probe the axial symmetry restoration that emerges in various contexts [66-70]. We leave these projects to near-future studies.

\section{ACKNOWLEDGMENTS}

The authors acknowledge Navid Abbasi, Marco Frasca, and John Petrucci for inspiration, discussions, and comments on the first version of this article. M. R. is supported by the National Science Foundation of China (Grants No. 11805087 and No. 11875153) and by the Fundamental Research Funds for the Central Universities (Grant No. 862946). M. C. is partially supported by Grant No. 0657-2020-0015 of the Ministry of Science and Higher Education of Russia. Z. Y. L. is supported by the Scientific Research Fund of Hunan Provincial Education Department (Grant No. 19C0772) and by the National Science Foundation of China (Grant No. 11835015).
[1] M. A. Shifman, Anomalies and low-energy theorems of quantum chromodynamics, Phys. Rep. 209, 341 (1991).

[2] T. Schäfer, Instantons and the Large N(c) Limit, A.D. 2004 (World Scientific, Singapore, 2005), pp. 28-45.

[3] D. E. Kharzeev, L. D. McLerran, and H. J. Warringa, The effects of topological charge change in heavy ion collisions: 'Event by event $\mathrm{P}$ and $C P$ violation', Nucl. Phys. A803, 227 (2008).

[4] K. Fukushima, D. E. Kharzeev, and H. J. Warringa, The Chiral magnetic effect, Phys. Rev. D 78, 074033 (2008).
[5] K. Fukushima, D. E. Kharzeev, and H. J. Warringa, RealTime Dynamics of the Chiral Magnetic Effect, Phys. Rev. Lett. 104, 212001 (2010).

[6] K. Fukushima, D. E. Kharzeev, and H. J. Warringa, Electriccurrent susceptibility and the Chiral magnetic effect, Nucl. Phys. A836, 311 (2010).

[7] K. Fukushima, M. Ruggieri, and R. Gatto, Chiral magnetic effect in the PNJL model, Phys. Rev. D 81, 114031 (2010).

[8] K. Fukushima and M. Ruggieri, Dielectric correction to the Chiral magnetic effect, Phys. Rev. D 82, 054001 (2010). 
[9] D. E. Kharzeev, The Chiral magnetic effect and anomalyinduced transport, Prog. Part. Nucl. Phys. 75, 133 (2014).

[10] V. Rubakov, On chiral magnetic effect and holography, arXiv:1005.1888.

[11] A. Gynther, K. Landsteiner, F. Pena-Benitez, and A. Rebhan, Holographic anomalous conductivities and the Chiral magnetic effect, J. High Energy Phys. 02 (2011) 110.

[12] K. Landsteiner, E. Megias, and F. Pena-Benitez, Anomalous transport from Kubo formulae, Lect. Notes Phys. 871, 433 (2013).

[13] M. M. Vazifeh and M. Franz, Electromagnetic Response of Weyl Semimetals, Phys. Rev. Lett. 111, 027201 (2013).

[14] N. Yamamoto, Generalized Bloch theorem and chiral transport phenomena, Phys. Rev. D 92, 085011 (2015).

[15] M. A. Zubkov, Absence of equilibrium chiral magnetic effect, Phys. Rev. D 93, 105036 (2016).

[16] M. Ruggieri, G. X. Peng, and M. Chernodub, Chiral relaxation time at the crossover of quantum chromodynamics, Phys. Rev. D 94, 054011 (2016).

[17] C. Manuel and J. M. Torres-Rincon, Dynamical evolution of the chiral magnetic effect: Applications to the quark-gluon plasma, Phys. Rev. D 92, 074018 (2015).

[18] V. P. Pagura, D. G. Dumm, S. Noguera, and N. N. Scoccola, Magnetic catalysis and inverse magnetic catalysis in nonlocal chiral quark models, Phys. Rev. D 95, 034013 (2017).

[19] S. P. Klevansky, The Nambu-Jona-Lasinio model of quantum chromodynamics, Rev. Mod. Phys. 64, 649 (1992).

[20] T. Hell, S. Rossner, M. Cristoforetti, and W. Weise, Dynamics and thermodynamics of a non-local PNJL model with running coupling, Phys. Rev. D 79, 014022 (2009).

[21] Y. Nambu and G. Jona-Lasinio, Dynamical model of elementary particles based on an analogy with superconductivity. 1, Phys. Rev. 122, 345 (1961).

[22] Y. Nambu and G. Jona-Lasinio, Dynamical model of elementary particles based on an snalogy with superconductivity. II, Phys. Rev. 124, 246 (1961).

[23] T. Hatsuda and T. Kunihiro, QCD phenomenology based on a chiral effective Lagrangian, Phys. Rep. 247, 221 (1994).

[24] M. Buballa, NJL model analysis of quark matter at large density, Phys. Rep. 407, 205 (2005).

[25] S. M. Schmidt, D. Blaschke, and Y. L. Kalinovsky, Scalarpseudoscalar meson masses in nonlocal effective QCD at finite temperature, Phys. Rev. C 50, 435 (1994).

[26] R. D. Bowler and M. C. Birse, A nonlocal, covariant generalization of the NJL model, Nucl. Phys. A582, 655 (1995).

[27] R.S. Plant and M.C. Birse, Meson properties in an extended nonlocal NJL model, Nucl. Phys. A628, 607 (1998).

[28] D. Blaschke, G. Burau, Y. L. Kalinovsky, P. Maris, and P. C. Tandy, Finite $\mathrm{T}$ meson correlations and quark deconfinement, Int. J. Mod. Phys. A 16, 2267 (2001).

[29] D. Gomez Dumm, D. B. Blaschke, A. G. Grunfeld, and N. N. Scoccola, Phase diagram of neutral quark matter in nonlocal chiral quark models, Phys. Rev. D 73, 114019 (2006).

[30] M. Frasca, Chiral symmetry in the low-energy limit of QCD at finite temperature, Phys. Rev. C 84, 055208 (2011).

[31] M. Frasca, $\rho$ condensation and physical parameters, J. High Energy Phys. 11 (2013) 099.
[32] T. Hell, S. Rossner, M. Cristoforetti, and W. Weise, Thermodynamics of a three-flavor nonlocal PolyakovNambu-Jona-Lasinio model, Phys. Rev. D 81, 074034 (2010).

[33] K. Langfeld and C. Kettner, The quark condensate in the GMOR relation, Mod. Phys. Lett. A 11, 1331 (1996).

[34] K. Langfeld, C. Kettner, and H. Reinhardt, A renormalizable extension of the NJL model, Nucl. Phys. A608, 331 (1996).

[35] M. Ruggieri and G. X. Peng, Critical temperature of Chiral symmetry restoration for quark matter with a Chiral chemical potential, J. Phys. G 43, 125101 (2016).

[36] M. Frasca, Nonlocal Nambu-Jona-Lasinio model and chiral chemical potential, Eur. Phys. J. C 78, 790 (2018).

[37] M. Ruggieri, The critical end point of quantum chromodynamics detected by Chirally imbalanced quark matter, Phys. Rev. D 84, 014011 (2011).

[38] M. N. Chernodub and A. S. Nedelin, Phase diagram of chirally imbalanced QCD matter, Phys. Rev. D 83, 105008 (2011).

[39] R. Gatto and M. Ruggieri, Hot quark matter with an axial chemical potential, Phys. Rev. D 85, 054013 (2012).

[40] L. Yu, H. Liu, and M. Huang, Effect of the chiral chemical potential on the chiral phase transition in the NJL model with different regularization schemes, Phys. Rev. D 94, 014026 (2016).

[41] R. L. S. Farias, D. C. Duarte, G. Krein, and R. O. Ramos, Thermodynamics of quark matter with a chiral imbalance, Phys. Rev. D 94, 074011 (2016).

[42] N. Y. Astrakhantsev, V. V. Braguta, A. Y. Kotov, and A. A. Nikolaev, Lattice study of QCD at finite chiral density: Topology and confinement, arXiv:1902.09325.

[43] V. V. Braguta, E. M. Ilgenfritz, A. Y. Kotov, B. Petersson, and S. A. Skinderev, Study of QCD phase diagram with non-zero chiral chemical potential, Phys. Rev. D 93, 034509 (2016).

[44] V. V. Braguta, V. A. Goy, E.-M. Ilgenfritz, A. Y. Kotov, A. V. Molochkov, M. Muller-Preussker, and B. Petersson, Twocolor QCD with non-zero Chiral chemical potential, J. High Energy Phys. 06 (2015) 094.

[45] S. S. Xu, Z. F. Cui, B. Wang, Y. M. Shi, Y. C. Yang, and H. S. Zong, Chiral phase transition with a chiral chemical potential in the framework of Dyson-Schwinger equations, Phys. Rev. D 91, 056003 (2015).

[46] B. Wang, Y. L. Wang, Z. F. Cui, and H. S. Zong, Effect of the chiral chemical potential on the position of the critical endpoint, Phys. Rev. D 91, 034017 (2015).

[47] C. Shi, X. He, W. Jia, Q. Wang, S. Xu, and H. Zong, Chiral transition and the chiral charge density of the hot and dense QCD matter, J. High Energy Phys. 06 (2020) 122.

[48] M. Ruggieri and G.X. Peng, Quark matter in a parallel electric and magnetic field background: Chiral phase transition and equilibration of chiral density, Phys. Rev. D 93, 094021 (2016).

[49] L. Yu, J. Van Doorsselaere, and M. Huang, Inverse magnetic catalysis in the three-flavor NJL model with axial-vector interaction, Phys. Rev. D 91, 074011 (2015).

[50] M. Hanada and N. Yamamoto, Universality of phase diagrams in QCD and QCD-like theories, Proc. Sci., LATTICE2011 (2011) 221 [arXiv:1111.3391]. 
[51] D. Ebert, T. G. Khunjua, K. G. Klimenko, and V.C. Zhukovsky, Competition and duality correspondence between chiral and superconducting channels in $(2+1)$ dimensional four-fermion models with fermion number and chiral chemical potentials, Phys. Rev. D 93, 105022 (2016).

[52] S. S. Afonin, A. A. Andrianov, and D. Espriu, The masses of vector mesons in holographic QCD at finite chiral chemical potential, Phys. Lett. B 745, 52 (2015).

[53] A. A. Andrianov, D. Espriu, and X. Planells, An effective QCD Lagrangian in the presence of an axial chemical potential, Eur. Phys. J. C 73, 2294 (2013).

[54] X. Planells, A. A. Andrianov, V. A. Andrianov, and D. Espriu, An effective theory for QCD with an axial chemical potential, Proc. Sci., QFTHEP2013 (2013) 049 [arXiv:1310 .4434].

[55] Z. Y. Lu and M. Ruggieri, Effect of the chiral phase transition on axion mass and self-coupling, Phys. Rev. D 100, 014013 (2019).

[56] H. D. Politzer, Effective quark masses in the Chiral limit, Nucl. Phys. B117, 397 (1976).

[57] V. V. Braguta and A. Y. Kotov, Catalysis of dynamical Chiral symmetry breaking by Chiral chemical potential, Phys. Rev. D 93, 105025 (2016).

[58] C. Gattringer, R. Hoffmann, and S. Schaefer, The topological susceptibility of SU(3) gauge theory near T(c), Phys. Lett. B 535, 358 (2002).

[59] G. Cossu, S. Aoki, H. Fukaya, S. Hashimoto, T. Kaneko, H. Matsufuru, and J. I. Noaki, Finite temperature study of the axial $U(1)$ symmetry on the lattice with overlap fermion formulation, Phys. Rev. D 87, 114514 (2013).

[60] H. T. Ding, Lattice QCD at nonzero temperature and density, Proc. Sci., LATTICE2016 (2017) 022 [arXiv: 1702.00151].
[61] S. Borsanyi, Z. Fodor, J. Guenther, K. Kampert, S. Katz, T. Kawanai, T. Kovacs, S. Mages, A. Pasztor, F. Pittler, J. Redondo, A. Ringwald, and K. Szabo, Calculation of the axion mass based on high-temperature lattice quantum chromodynamics, Nature (London) 539, 69 (2016).

[62] A. Das, D. Kumar, and H. Mishra, Chiral susceptibility in the NambuJona-Lasinio model: A Wigner function approach, Phys. Rev. D 100, 094030 (2019).

[63] M. Ruggieri, Z. Y. Lu, and G. X. Peng, Influence of chiral chemical potential, parallel electric, and magnetic fields on the critical temperature of QCD, Phys. Rev. D 94, 116003 (2016).

[64] A. Y. Kotov, M. P. Lombardo, and A. M. Trunin, Fate of the $\eta^{\prime}$ in the quark gluon plasma, Phys. Lett. B 794, 83 (2019).

[65] A. Bazavov et al. (HotQCD Collaboration), Chiral crossover in QCD at zero and non-zero chemical potentials, Phys. Lett. B 795, 15 (2019).

[66] L. He, M. Jin, and P. Zhuang, Effect of U(A)(1) breaking on chiral phase structure and pion superfluidity at finite isospin chemical potential, Mod. Phys. Lett. A 22, 637 (2007).

[67] L. Wang, G. Cao, X. Huang, and P. Zhuang, NambuJonaLasinio model in a parallel electromagnetic field, Phys. Lett. B 780, 273 (2018).

[68] A. Bazavov et al. (HotQCD Collaboration), The chiral transition and $U(1)_{A}$ symmetry restoration from lattice QCD using domain wall fermions, Phys. Rev. D 86, 094503 (2012).

[69] J. I. Kapusta, E. Rrapaj, and S. Rudaz, Is hyperon polarization in relativistic heavy ion collisions connected to axial U(1) symmetry breaking at high temperature?, Phys. Rev. C 101, 031901 (2020).

[70] K. Suzuki et al. (JLQCD Collaboration), Axial U(1) symmetry, topology, and Dirac spectra at high temperature in $N_{f}=2$ lattice QCD, Proc. Sci., CD2018 (2019) 085 [arXiv:1908.11684]. 